\title{
CONTROL TENSIONAL A CORTO Y MEDIO PLAZO DE FACOESCLERECTOMÍA PROFUNDA NO PERFORANTE SIN IMPLANTE ESCLERAL NI ANTIMETABOLITO
}

\author{
SHORT- AND MEDIUM-TERM INTRAOCULAR PRESSURE \\ LOWERING EFFECTS OF COMBINED PHACOEMULSIFICATION \\ AND NON-PENETRATING DEEP SCLERECTOMY WITHOUT \\ SCLERAL IMPLANT OR ANTIFIBROTICS
}

\author{
MORENO-LÓPEZ M ${ }^{1}$, PÉREZ-ÁLVAREZ MJ ${ }^{1}$
}

\begin{abstract}
RESUMEN
Objetivo: Evaluar los resultados sobre la presión intraocular (PIO) a corto y medio plazo de la cirugía combinada de facoemulsificación y esclerectomía profunda no perforante (FEPNP) sin implante escleral ni antimetabolito en el glaucoma de ángulo abierto, primario y pseudoexfoliativo (GAA).

Métodos: Estudio retrospectivo de quince ojos de doce pacientes con GAA mal controlado farmacológicamente con catarata o que, estando bien controlados con medicación, precisaban ser intervenidos de catarata. Todos fueron intervenidos de FEPNP por un mismo cirujano. La PIO postoperatoria por encima del objetivo preestablecido fue tratada mediante punción de membrana trabéculo-descemética con láser Nd-YAG y/o manipulación de la ampolla de filtración con mitomicina C. Los principales parámetros evaluados fueron: PIO pre y postoperatoria, porcentaje de ojos con PIO $<17 \mathrm{mmHg}$, reducción en número de fármacos hipotensores, incidencia de complicaciones y agudeza visual (AV). El seguimiento mediano fue de 12,00 meses (DE: 0,6$)$ con un rango de 1 a 30 meses.

Resultados: La PIO preoperatoria media era 21,80 (DE: 5,14 ) y descendió a 14,42 (DE: 2,15 ) a los 12

Recibido: 23/6/04. Aceptado: 8/2/06

Servicio de Oftalmología. Hospital Universitario de Guadalajara. España.

1 Licenciado en Medicina.

Correspondencia:

María Moreno López

C/. Dalí, 14

19171 Cabanillas del Campo (Guadalajara)

España

E-mail: mqmorenol@yahoo.es
\end{abstract}

\begin{abstract}
Purpose: To examine the short- and medium-term intraocular pressure (IOP) lowering effects of combined phacoemulsification and non-penetrating deep sclerectomy without the use of scleral implant or antifibrotics in open-angle glaucoma (primary and pseudoexfoliative) and coexisting cataract in eyes with no known risk factors for bleb failure.

Methods: Retrospective study of 15 eyes of 12 patients with medically uncontrolled open-angle glaucoma or open-angle glaucoma treated with two or more drugs and coexisting cataract with no known risk factors for glaucoma surgery failure. All patients received combined phacoemulsification and nonpenetrating deep sclerectomy without scleral implant or antifibrotics performed by the same surgeon. NdYAG perforation of the trabeculodescemetic membrane and/or needling with mitomycin-C was performed postoperatively for IOP control. Main outcome measures were postoperative IOP, percentage of eyes with IOP $<17 \mathrm{mmHg}$, complications and final visual acuity (VA). Median follow-up was 12.0 months (SD: 0.6) and ranged from 1 to 30 months.
\end{abstract}

Results: Mean preoperative IOP with medical treatment was $21.80 \mathrm{mmHg}$ (SD: 5.14) and decreased 
meses. La media de fármacos hipotensores empleados en el preoperatorio fue 1,97 (DE: 0,70) y descendió a 0,13 (DE: 0,35) tras cirugía. A los 12 meses, el 80\% mantienen $\mathrm{PIO}<17 \mathrm{mmHg}$ con una ganancia media de AV de 2,50 (DE: 1,91) líneas de Snellen. La complicación postoperatoria más frecuente fue la filtración a través del colgajo conjuntival, ocurrida en tres de los quince ojos (20\%).

Conclusiones: La FEPNP sin implante ni antimetabolito es una opción efectiva en el manejo del GAA con catarata en ojos sin factores de riesgo de fracaso de cirugía filtrante. Esta técnica reduce significativamente la PIO así como la necesidad de fármacos antiglaucomatosos a corto y medio plazo permitiendo una rápida rehabilitación visual con escasas complicaciones.

Palabras claves: Esclerectomía profunda sin implante, facoemulsificación, glaucoma. to $14.42 \mathrm{mmHg}$ (SD: 2.15) at 12-month visit. Mean antiglaucoma medication preoperative was 1.93 (SD: 0.70) and was reduced to 0.13 (DE: 0.35) postoperative. At 12-month visit, $80 \%$ had an IOP lower than $17 \mathrm{mmHg}$ with a mean VA gain of 2.50 Snellen lines. Conjuntival wound leakage was the most frequent complication $(20 \% ; 3 / 15)$.

Conclusions: Primary combined phacoemulsification and non-penetrating deep sclerectomy without collagen implant or antifibrotics in primary openangle glaucoma with coexisting cataract, significantly lowers IOP in the short- and medium term in low-risk cases for glaucoma surgery failure, allowing for rapid visual improvement with a low complication rate (Arch Soc Esp Oftalmol 2006; 81: 93100).

Key words: Deep sclerectomy without scleral implant, phacoemulsification, glaucoma.

\section{INTRODUCCIÓN}

La incidencia de GAA aumenta con la edad, lo que hace que con frecuencia coexista una catarata. No hay consenso sobre el manejo de estas dos patologías en el tiempo (1), sin embargo cada vez más cirujanos propugnan la combinación de ambos procedimientos como técnica que disminuye satisfactoriamente los niveles de PIO al tiempo que acelera la rehabilitación visual y reduce costes al evitar una segunda cirugía $(2,3)$.

La esclerectomía profunda no perforante (EPNP), aunque no es superior a la trabeculectomía en el control de la PIO, reduce las complicaciones tanto intraoperatorias como postoperatorias, y es en la actualidad una técnica quirúrgica en expansión (4-7). El uso de implantes esclerales parece aumentar la filtración y reducir la fibrosis de la ampolla pero encarece la cirugía, lo que en determinadas situaciones podría limitar su elección (8).

Los antimetabolitos, moduladores de la respuesta cicatricial, se emplean en un intento de aumentar la tasa de éxito, no estando exentos de complicaciones. La evidencia sugiere un beneficio a favor de la aplicación de mitomicina $\mathrm{C}$ como coadyuvante en la cirugía combinada de glaucoma y catarata, especialmente en pacientes con alto riesgo de fracaso (9).

Son factores de riesgo conocidos de fracaso de la cirugía filtrante determinadas enfermedades oculares como las inflamaciones y las cirugías oculares previas, el uso prolongado de medicación tópica antiglaucomatosa, la raza negra y la edad joven (9).

En nuestro conocimiento no hay estudios publicados sobre los resultados a corto y medio plazo de la combinación de facoemulsificacion y EPNP sin implante escleral ni antimetabolitos en el GAA sin factores de riesgo conocidos de fracaso de cirugía filtrante.

\section{SUJETOS, MATERIAL Y MÉTODOS}

Se seleccionaron pacientes diagnosticados de GAA (primario o pseudoexfoliativo) con catarata coexistente, cuya PIO no podía ser controlada adecuadamente con tratamiento farmacológico máximo (dos o más fármacos hipotensores oculares) o que debido a la catarata, precisaban ser intervenidos quirúrgicamente. Todos los casos fueron intervenidos de FEPNP sin implante escleral ni antimetabolito por un mismo cirujano, entre noviembre $2001 \mathrm{y}$ abril 2004 en nuestro centro, siguiendo la técnica descrita por Mermoud (10). Los criterios de exclusión fueron edad joven, raza negra, enfermedad inflamatoria ocular, cirugía ocular o láser previo. El rango de seguimiento fue de 1 a 30 meses, doce de los quince casos $(80 \%)$ tuvieron un seguimiento mínimo de 12 meses.

Los datos recogidos preoperatoriamente incluyeron: edad, género, tipo de glaucoma, PIO basal 
(preoperatorio sin tratamiento farmacológico hipotensor) medido con tonómetro de Goldmann, agudeza visual mejor corregida (AVMC), número de fármacos y tiempo de tratamiento tópico preoperatorio, PIO preoperatoria (en tratamiento farmacológico) y daño campimétrico. El campo visual fue analizado mediante perimetría estática computerizada (programa 30-2 del Zeiss-Humphrey Systems, Dublín,CA, Modelo 745i) preoperatoriamente evaluando el daño glaucomatoso siguiendo la clasificación establecida por Hodapp, Parish y Anderson (11).

La cirugía fue practicada por un mismo cirujano bajo anestesia retrobulbar ( $4 \mathrm{ml}$ de mezcla de bupivacaína $0,5 \%$ y lidocaína al $2 \%$ ) en quince ojos de doce pacientes. El procedimiento filtrante se realizó a las 12 horas tras un punto de tracción bajo el músculo recto superior, disección conjuntival base en fornix, diatermia de vasos sangrantes, disección de tapete escleral superficial de un tercio de espesor de $5 \times 5 \mathrm{~mm}$ entrando $1 \mathrm{~mm}$ en córnea clara y marcado de tapete escleral profundo. A continuación se procedía a realizar la facoemulsificación del cristalino a través de una incisión en córnea clara temporal en ojos derechos y nasal en ojos izquierdos y, tras introducir la lente intraocular, se cerraba la incisión con un punto en cruz de Nylon 10/0. Finalizada la facoemulsificación se procedía al tallado del tapete escleral profundo, canal de Schlemm y membrana de Descemet, resecando al final el colgajo profundo y realizando la trabeculectomía ab externo con pinzas de capsulorrexis. En caso de no observarse suficiente filtración a través de la membrana trabéculo-descemética creada, se «raspaba» dicha membrana con hemostetas secas con el objeto de eliminar fibras de colágeno no visibles que pudieran quedar y/o crear microtraumatismos que favorezcan la filtración. El colgajo superficial se suturaba con dos puntos sueltos de Nylon 10/0 cuya holgura permitiera introducir bajo el tapete un viscoelástico (Viscoat) hasta darle una forma de cúpula. La conjuntiva también se suturaba con puntos sueltos de Nylon 10/0.

En el postoperatorio, los pacientes fueron tratados con una mezcla de dexametasona y gentamicina o cloranfenicol (Colircusi De Icol ${ }^{\circledR}$ o Colircusi Gentadex $^{\circledR}$, ALCON) 6 veces al día durante la primera semana, con pauta descendente a razón de una gota a la semana hasta su suspensión.

Las visitas postoperatorias se realizaron, como mínimo, a las 24 horas, a la semana, al mes, cada 3 meses durante el primer año, y cada 6 meses en ade- lante. En cada visita se recogían los siguientes datos: AVMC, exploración biomicroscópica, PIO, número y tipo de fármacos hipotensores e incidencia y severidad de cualquier complicación postoperatoria.

Tras una buena respuesta tensional inicial, toda PIO superior a $18 \mathrm{mmHg}$ en cualquier visita postoperatoria fue considerada fracaso por insuficiente filtración, y tratada con goniopunción con láser NdYAG de la porción anterior de la membrana trabéculo-descemética, utilizando lente de Goldmann y/o manipulación de la ampolla de filtración con mitomicina C. El intervalo de tiempo entre FEPNP y cualquier tratamiento posterior fue registrado, así como los valores de PIO previos y a las 24 horas.

Al incluir en el estudio a pacientes con daño glaucomatoso avanzado, se definió éxito absoluto a una PIO $<17 \mathrm{mmHg}$ sin tratamiento médico y éxito relativo a una PIO $<17 \mathrm{~mm} \mathrm{Hg}$ con o sin tratamiento médico. Además se evaluaron el número de casos que lograron mantener una PIO $<21 \mathrm{mmHg}$ sin medicación y una PIO $<21 \mathrm{mmHg}$ con y sin fármacos antiglaucoma para ofrecer resultados comparables con otros estudios.

El análisis estadístico se realizó mediante el programa SPSS11.0 (SPSS inc., Chicago, Estados Unidos). Los datos cuantitativos de las variables a estudio se presentan como media y desviación estándar (DE). Se emplea el test estadístico t de Student para comparar dichas medias. Se considera significación estadística si $\mathrm{p}<0,05$. Para comparar el número de fármacos antiglaucoma empleados pre y postoperatorio se emplea el test no paramétrico de Wilcoxon. Debido a las diferencias en el seguimiento se realizan curvas de supervivencia de Kaplan-Meier. El tiempo de seguimiento se expresa en esta curva como mediana $\pm \mathrm{DE}$.

\section{RESULTADOS}

Se incluyen en el estudio quince ojos de doce pacientes. Cinco ojos correspondían a hombres $(33,3 \%)$ y diez ojos a mujeres $(66,6 \%)$. En diez casos $(66,6 \%)$ el diagnóstico fue glaucoma primario de ángulo abierto (GPAA) y en cinco $(33,3 \%)$, glaucoma pseudoexfoliativo (GPX). El rango de edad osciló entre 69 y 88 años, con una media de 81,07 años (DE: 4,97) (tabla I). El seguimiento mediano fue de 12,00 meses (DE: 0,62) con un rango entre 1 y 30 meses. El $80 \%$ de los casos (doce de 
Tabla I. Características demográficas de los casos

\begin{tabular}{|c|c|}
\hline $\mathrm{N}^{\circ}$ de pacientes & 12 \\
\hline $\mathrm{N}^{\mathrm{o}}$ de ojos & 15 \\
\hline $\begin{array}{l}\text { Edad (media) } \\
\quad(\text { rango) }\end{array}$ & $\begin{array}{l}\text { 81,07 (DE: 4,97) } \\
(69-88)\end{array}$ \\
\hline \multicolumn{2}{|l|}{ Sexo } \\
\hline Varones & 5 \\
\hline Mujeres (*) & 7 \\
\hline \multicolumn{2}{|l|}{ Tipo de glaucoma } \\
\hline GPAA & 10 \\
\hline Pseudoexfoliativo & 5 \\
\hline \multicolumn{2}{|l|}{ Grado severidad } \\
\hline Leve & $0(0 \%)$ \\
\hline Moderado & $6(40 \%)$ \\
\hline Severo & $9(60 \%)$ \\
\hline $\begin{array}{l}\text { PIO basal preoperatorio }(\mathrm{mmHg}) \\
\text { (media) }\end{array}$ & 33,69 (DE:10,57) \\
\hline $\begin{array}{l}\text { PIO preoperatorio en tratamiento } \\
(\mathrm{mmHg}) \text { (media) }\end{array}$ & 21,80 (DE:4,14) \\
\hline $\begin{array}{l}\text { AV preoperatorio } \\
(\text { media })(* *)\end{array}$ & 0,37 (DE: 0,11 ) \\
\hline $\begin{array}{l}\mathrm{N}^{\circ} \text { fármacos preoperatorio } \\
\text { (rango) }\end{array}$ & $\begin{array}{l}1,93(\mathrm{DE}: 0,7) \\
(1-4)\end{array}$ \\
\hline
\end{tabular}

*: en 3 mujeres fueron incluidos ambos ojos en el estudio; **: excluidos 3 ojos con AV no cuantificables ( 2 ojos = movimiento de manos, 1 ojo = cuenta dedos).

quince ojos) fueron seguidos durante 1 año, sólo cuatro de los quince ojos $(26,66 \%)$ tuvieron un seguimiento de 18 meses, dos ojos fueron seguidos durante 2 años y un ojo durante 30 meses. Ningún paciente dejó de acudir a sus visitas postoperatorias (tabla II).

Evaluado el grado de daño glaucomatoso, seis de los quince ojos (40\%) presentaban daño moderado y nueve de los quince ojos (60\%) presentaban un daño severo (tabla I).
La PIO basal media fue de 33,69 mmHg (DE: 10,57). La PIO preoperatoria media fue de 21,80 mmHg (DE: 5,14). El tiempo medio de duración del tratamiento tópico hipotensor previo a la cirugía fue de 21,2 meses (DE: 13,34), sólo un paciente (dos ojos) mantuvo tratamiento preoperatorio durante más de 3 años. La PIO postoperatoria media a las 24 horas fue de $6,85 \mathrm{mmHg}$ (DE: 7,17), al mes $15,40 \mathrm{mmHg}$ (DE: 3,76), a los 3 meses $15 \mathrm{mmHg}$ (DE: 3,86), a los 6 meses 13, $76 \mathrm{mmHg}$ (DE: 2,20), a los 9 meses 14,33 mmHg (DE: 2,42), a los 12 meses 14,42 mmHg (DE: 2,15), a los 18 meses $14,24 \mathrm{mmHg}$ (DE: 2,21), a los 24 meses $16 \mathrm{mmHg}$ (DE: 1,41), el único paciente cuyo seguimiento fue de 30 meses presentó una PIO de $17 \mathrm{mmHg}$ en la última visita (fig. 1). Hubo una reducción estadísticamente significativa $(\mathrm{p}<0,05)$ entre la PIO postoperatoria y la PIO preoperatorio en todas las visitas

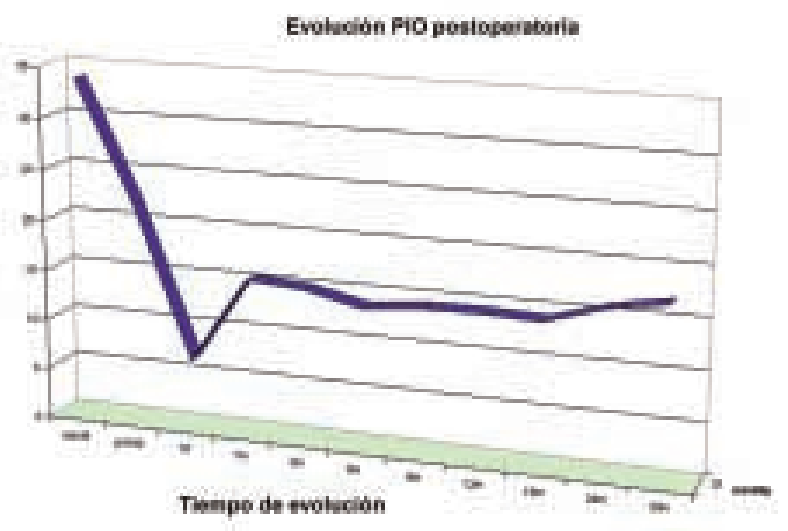

Fig. 1: Evolución de la PIO postoperatoria.

Tabla II. Resultados tensionales y seguimiento

\begin{tabular}{|c|c|c|c|c|c|}
\hline \multirow[b]{2}{*}{ PIO basal } & \multirow{2}{*}{$\begin{array}{l}\mathrm{N} \\
15\end{array}$} & \multicolumn{2}{|c|}{ PIO (mmHg) } & $\begin{array}{c}\text { Reducción media PIO } \\
\text { vs PIO preop. }\end{array}$ & \multirow[t]{2}{*}{$\mathrm{P}$} \\
\hline & & 33,69 & (DE: 10,57) & & \\
\hline PIO $\mathrm{tt}^{\circ}$ preop. & 15 & 21,80 & (DE: 5,14$)$ & & \\
\hline \multicolumn{6}{|l|}{ PIO posop. } \\
\hline 1 día & 15 & 6,85 & (DE: 7,17) & 15,30 (DE: 8,06) & $<0,01$ \\
\hline 1 mes & 15 & 15,40 & (DE: 3,76) & $6,40 \quad(\mathrm{DE}: 5,70)$ & $<0,01$ \\
\hline 3 mes & 14 & 15 & (DE: 3,86) & 6,28 (DE: 6,95 ) & \\
\hline 6 mes & 13 & 13,77 & (DE: 2,20) & $6,46$ (DE: 4,05$)$ & $<0,01$ \\
\hline 9 mes & 12 & 14,33 & (DE: 2,42) & $6,16$ (DE: 5,16$)$ & \\
\hline 12 mes & 12 & 14,42 & (DE: 2,15) & $5,75$ (DE: 4,43$)$ & $<0,01$ \\
\hline 18 mes & 4 & 14,24 & (DE: 2,21) & $4,50$ (DE: 3,31$)$ & \\
\hline 24 mes & 2 & 16 & (DE: 1,41) & (DE: 2,82) & \\
\hline 30 mes & 1 & 17 & & (DE: 0) & \\
\hline
\end{tabular}

PIO tt ${ }^{\circ}$ preop.: presión intraocular preoperatorio con tratamiento; PIO posop.: presión intraocular posoperatoria. 
hasta la visita del mes 12 , no pudiendo sacar conclusiones más allá de este periodo.

La media de fármacos hipotensores preoperatorios fue 1,93 (DE: 0,70; rango: 1-3) que se redujo a una media de 0,13 (DE: 0,35 ) a los 12 meses de la cirugía con una reducción estadísticamente significativa del número de fármacos empleados en todas las visitas postoperatorias $(\mathrm{p}<0,05)$. Sólo dos ojos precisaron tratamiento hipotensor para mantener PIO por debajo de $17 \mathrm{mmHg}$, el primero a las 6 semanas como tratamiento médico de un quiste de tenon y el segundo caso a los 12 meses.

Según la curva de supervivencia de KaplanMeier, se obtuvo éxito tanto absoluto como relativo (PIO $<17 \mathrm{mmHg}$ sin tratamiento y $\mathrm{PIO}<17 \mathrm{mmHg}$ con y sin tratamiento) en el $86,61 \%$ y $80 \%$ a los 6 y 12 meses de seguimiento respectivamente. En estos períodos, las cifras no varían si consideramos éxito como PIO <21 mmHg. Dado que el tiempo mediano de seguimiento es de 12 meses, no podemos sacar conclusiones por el momento más allá de este periodo (fig. 2).

La goniopunción con láser Nd-YAG de la membrana trabéculo-descemética fue practicada en cuatro casos (26,66\%), al mes, dos ojos y a los 4 y 9 meses los otros dos ojos, uno de los cuales requirió además manipulación de la ampolla de filtración con mitomicina $\mathrm{C}$ a los 3 meses de la goniopunción.

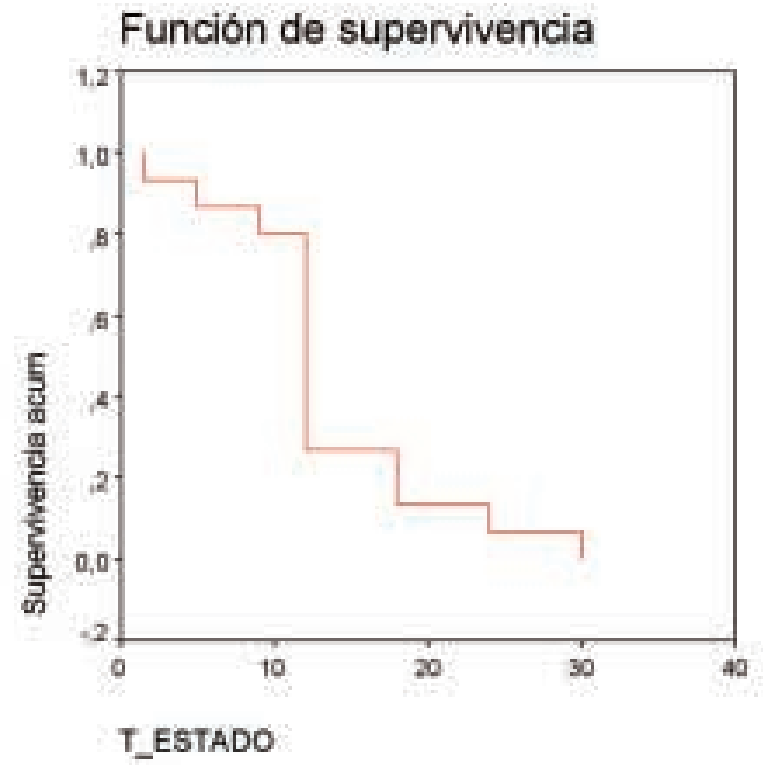

Fig. 2: Curva de supervivencia de Kaplan-Meier. Éxito absoluto (PIO $<17 \mathrm{mmHg}$ sin tratamiento).

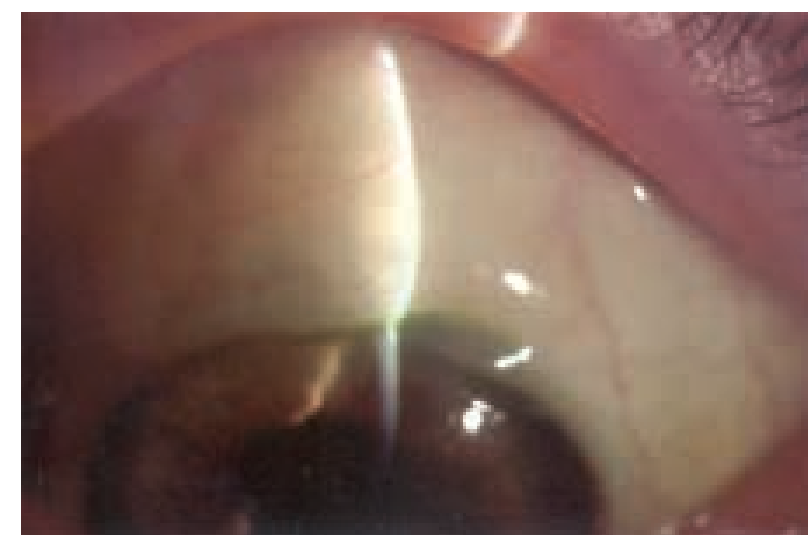

Fig. 3: «Buena» ampolla de filtración a los 12 meses.

La PIO media previa a la goniopunción fue de 20mmHg (rango: 18-24), y tras el láser de 14,5 mmHg (rango: 13-18) siendo el descenso medio de $5,5 \mathrm{mmHg}$. En nuestra serie se puede considerar exitosa la goniopunción en tres de los cuatro ojos tratados (75\%), ya que un caso sólo obtuvo un descenso de $1 \mathrm{mmHg}$.

Entre las complicaciones intraoperatorias se encontraron una desinserción del espolón escleral y una rotura de la cápsula posterior. La complicación postoperatoria más frecuente fue la filtración a través del colgajo conjuntival (fenómeno de Seidel) que ocurrió en el 20\% (3/15), todos ellos resuelto con lente de contacto terapéutica. Otras complicaciones postoperatorias fueron un caso de hipotalamia, un resto de núcleo retenido inadvertidamente en cámara anterior que precisó su extracción quirúrgica y el desarrollo de un quiste de tenon a las 6 semanas de la intervención y que en el momento de

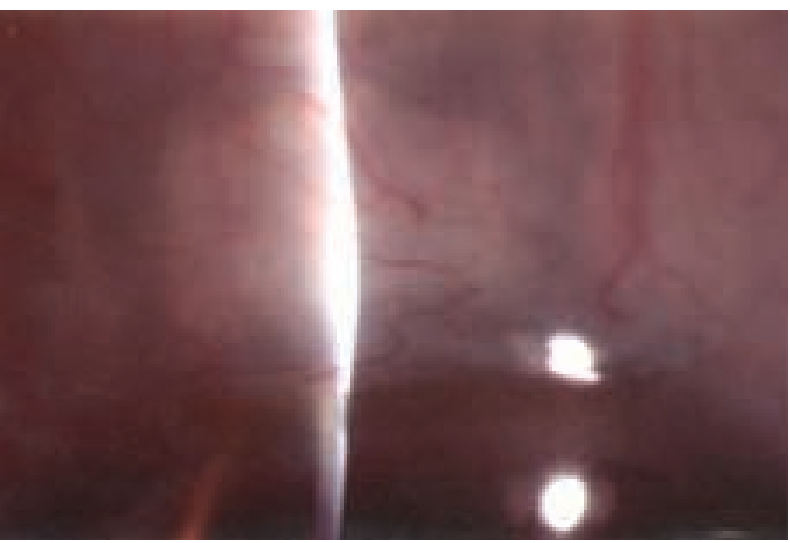

Fig. 4: Ampolla de filtración «plana»o «en regresión» a los 12 meses. 
escribir este artículo (5 meses) sigue en tratamiento con un fármaco. No hubo ningún caso de hifema, reacción inflamatoria severa, blebitis, endoftalmitis, desprendimiento coroideo ni de retina.

La AV prequirúrgica media, al excluir los tres ojos con visiones no cuantificables [dos ojos con movimientos de mano (MM) y un ojo con cuenta dedos (CD)], fue de 0,37 (DE: 0,11). La AV final media fue de 0,58 (DE: 0,18) con un rango que va de 0,25 a 0,9. La ganancia media en líneas de Snellen al final del seguimiento fue de 2,50 (DE: 1,91) con un $57,1 \%$ de casos (ocho de catorce ojos) que mejoran $\geq 2$ líneas de Snellen.

\section{DISCUSIÓN}

La cirugía filtrante no perforante no supera a la trabeculectomía convencional en términos de reducción de PIO, sin embargo minimiza la incidencia de complicaciones facilitando el manejo ambulatorio durante el postoperatorio inmediato, con buenos resultados tensionales (7,12-16). El uso de implantes esclerales intenta mejorar los resultados $(2,5,12,17)$ no sin un aumento significativo del coste, lo que en ocasiones limita su aplicación.

La cirugía de glaucoma suele ser poco gratificante para los pacientes al no verse acompañado de una mejoría de agudeza visual, pues éstos perciben como único beneficio la reducción de la medicación hipotensora. Asociando la facoemulsificación del cristalino opaco se logran resultados tensionales satisfactorios además de una rápida rehabilitación visual (15) aunque no existe consenso respecto al momento óptimo de su realización (1). La evidencia parece respaldar el uso de antimetabolitos en la cirugía combinada de catarata y glaucoma pues muchos autores la consideran en sí misma factor de riesgo de fracaso, no estando su uso exento de complicaciones (9).

Presentamos los resultados preliminares de la FEPNP sin implante escleral ni antimetabolito como tratamiento del GAA, primario y pseudoexfoliativo, en pacientes mal controlados farmacológicamente o que estando en tratamiento medicamentoso precisaban ser intervenidos de catarata. A excepción de dos ojos, un paciente, que había sido tratado con medicación tópica durante más de 3 años, nuestros casos no presentaban factores de riesgo de fracaso de cirugía filtrante añadidos a la cirugía combinada en sí.
En nuestro estudio se observa un descenso significativo de la PIO tras la cirugía. La reducción media de la PIO con respecto a la preoperatoria a los 6 y 12 fue de $6,46 \mathrm{mmHg}(\mathrm{DE}: 4,05)$ y 5,75 mmHg (DE: 4,43) (tabla II). Comparando con otros autores, Gianoli en sus resultados de FEPNP con implante de colágeno consigue una PIO media de 11,9 $\mathrm{mmHg}$ (DE: 3,6) a los 6 meses y de 13,9 mmHg (DE: 4,7) a los 12 meses (14), mientras que Muñoz-Negrete en un estudio retrospectivo de 39 ojos intervenidos mediante FEPNP con implante de ácido hialurónico reticulado, a partir de una PIO media prequirúrgica de 23,1 mmHg (DE: 8,9) logra una reducción media de PIO de 8,63 a los 6 meses y de 6,88 a los 12 meses, con una PIO media postoperatoria a los 12 meses de 15,66 (DE: 4,03) (3).

Como ya se ha apuntado durante el análisis de los resultados, se decidió establecer como criterio de éxito una PIO objetivo $<17 \mathrm{mmHg}$ dado que en nuestra casuística se incluyen hasta un $60 \%$ de casos con daño glaucomatoso severo, alcanzando según la curva de Kaplan-Meier, el éxito tanto absoluto como relativo en el $80 \%$ de los casos a los 12 meses.

Según los criterios clásicos tanto de éxito absoluto como relativo (PIO $<21 \mathrm{mmHg}$ sin tratamiento y PIO $<21 \mathrm{mmHg}$ con y sin tratamiento), se obtiene el mismo resultado al año, un $80 \%$ de éxito quirúrgico. En comparación con otros estudios, Gianoli (14) obtiene éxito absoluto en el 59\% y éxito relativo en el $88 \%$ de los pacientes a los 12 meses, y Muñoz-Negrete (3) describe un $67,6 \%$ de éxito absoluto y 94,1\% de éxito relativo a los 12 meses.

Con respecto a la reducción en fármacos hipotensores, el 93,33\% de nuestros casos a los 6 meses y el $86,66 \%$ a los 12 meses se mantenían libres de tratamiento, pero se debe recordar que esta cifra se verá previsiblemente reducida cuando se dispongan de los datos de seguimiento al año de los quince casos, ya que de momento sólo se disponen datos de doce pacientes. Estos resultados son comparables al $84,2 \%$ y $71,9 \%$ sin tratamiento a los 6 y 12 meses respectivamente publicados por Muñoz-Negrete (3).

La goniopunción con láser Nd-YAG de la membrana trabéculo-descemética fue realizada en el $26,66 \%$ de los pacientes, porcentaje que se sitúa dentro del rango descrito por otros autores, Mermoud la realiza en el $41 \%$ de los casos (8), Shaarawy en el 46\% (5), Manelli en el 24,2\% (18), Sánchez en el 15\% (17) y El-Sayyad en el 10,3\% (7). $\mathrm{El}$ intervalo tras la cirugía fue variable, 1,4 y 9 
meses, comparables al intervalo descrito en la literatura $(5,7,8,14,17,18)$. La PIO media antes de la goniopunción era de $20 \mathrm{mmHg}$ (DE: 2,70) y la PIO media posterior fue de 14,66 mmHg (DE: 2,88), lo que supone un descenso medio de $5,5 \mathrm{mmHg}$, cifras similares a las series de otros autores que oscilan entre 4 y $9,7 \mathrm{mmHg}$ de descenso tensional medio (18). La tasa de éxito inmediato fue del $75 \%$, tres pacientes lograron controlar su tensión intraocular tras la goniopunción y uno de ellos, el que consideramos fracaso, precisó revisión con aguja y mitomicina $\mathrm{C}$ de la ampolla de filtración. Los buenos resultados de la goniopunción son avalados por otros autores con altas tasas de éxito inmediato, así Mermoud describe un $83 \%$ de éxito (8). Sin embargo existe un porcentaje de casos que requieren revisión posterior de la ampolla para mantener el control tensional, Manelli precisó «needling» en el 12,1\% de los casos y revisión quirúrgica en el 6,1\% (18), El-Sayyad realiza inyecciones subconjuntivales de 5-fluouracilo en el 43,6\% de los pacientes (7).

Respecto a la AV corregida, se logró una ganancia media en líneas de Snellen de 2,50 (DE: 1,51), con una mejoría $\geq 2$ líneas de Snellen en un $57,1 \%$ de los ojos. La mejoría visual no suele producirse durante la primera semana postoperatoria debido a edema corneal, hipotalamia, suturas apretadas entre otras causas, estabilizándose a partir del mes. En nuestro estudio, la $\mathrm{AV}$ media preoperatorio fue de 0,37 (DE: 0,11) y al final del seguimiento fue de 0,58 (DE: 0,18), comparable con las encontradas por Gianoli (14) que describe una mejoría de AV de 0,18 preoperatoria a 0,5 tras cirugía y MuñozNegrete (3) que pasa de una AV corregida preoperatorio de 0,38 a una $\mathrm{AV}$ al año de 0,66.

No se produjeron complicaciones intraoperatorias severas. La complicación postoperatoria más frecuente fue la filtración a través del colgajo conjuntival $(20 \%)$ a diferencia de las encontradas por Muñoz-Negrete (3) que describe la reacción inflamatoria severa $(7,7 \%)$ e hifema $(5,1 \%)$ como las más frecuentes. El resto de complicaciones postoperatorias fueron raras. No hubo ningún caso de hifema, reacción inflamatoria severa, blebitis, endoftalmitis, desprendimiento coroideo ni de retina.

No hemos encontrado estudios sobre los resultados a corto y medio plazo de la combinación de FEPNP sin implante escleral ni antimetabolito en el GAA sin factores de riesgo conocidos de fracaso. A pesar de las limitaciones de nuestro estudio derivadas del carácter retrospectivo, reducida casuística, diferencias en el tiempo de seguimiento y ausencia de grupo control tanto con implante escleral como con antimetabolitos, nuestros resultados reflejados en cuanto a control de PIO, reducción de fármacos, rehabilitación visual y ausencia de complicaciones severas, nos hacen reflexionar sobre la necesidad de asociar implante escleral o antimetabolitos en este grupo de pacientes. Estudios futuros más amplios, con mayor seguimiento y grupo control nos ayudarán a definir esta técnica de cirugía combinada.

\section{BIBLIOGRAFÍA}

1. Baudouin $C$. When should glaucoma be surgically treated? J Fr Ophtalmol 2001; 24: 1103-1109.

2. Ates $H$, Andac $K$, Uretmen $O$. Non-penetrating deep sclerectomy and collagen implant surgery in glaucoma patients with advanced field loss. International Ophthalmology 2001; 23: 123-128.

3. Muñoz-Negrete FJ, Rebolleda G, Noval S. Non-penetrating deep sclerectomy combined with phacoemulsification. Results and complications. Arch Soc Esp Oftalmol 2003; 78: 499-506.

4. Mermoud A, Schnyder CC, Sickenberg M, Chiou AG, Hediguer SE, Faggioni R. Comparison of deep sclerectomy with collagen implant and trabeculectomy in open-angle glaucoma. J Cataract Refract Surg 1999; 25: 323-331.

5. Shaarawy T, Karlen M, Schnyder C, Achache F, Sanchez $E$, Mermoud A. Five-year results of deep sclerectomy with collagen implant. J Cataract Refract Surg 2001; 27: 17701778.

6. Massy J, Gruber D, Muraine M, Brasseur G. Non-penetrating deep sclerectomy in the treatment of chronic openangle glaucoma. Mid-term results. J Fr Ophtalmol 1999; 22: 292-298.

7. El Sayyad F, Helal M, El-Kholify H, Khalil M, El-Maghraby A. Nonpenetrating deep sclerectomy versus trabeculectomy in bilateral primary open-angle glaucoma. Ophthalmology 2000; 107: 1671-1674.

8. Mermoud A, Karlen ME, Schnyder CC, Sickenberg M, Chiou AG, Hediguer SE, et al. Nd: Yag goniopuncture after deep sclerectomy with collagen implant. Ophthalmic Surg Lasers 1999; 30: 120-125.

9. Lama PJ, Fechtner RD. Antifibrotics and wound healing in glaucoma surgery. Surv Ophthalmol 2003; 48: 314-346.

10. Mermoud A. La sclerectomie profonde. Technique chirurgicale. J Fr Ophtalmol 1999; 22: 781-786.

11. Hodapp E, Parrish RK, Anderson DR. Clinical decisions in glaucoma. St Louis: Mosby; 1993; 79.

12. Drolsum L. Conversion from trabeculectomy to deep sclerectomy. Prospective study of the first 44 cases. $J$ Cataract Refract Surg 2003; 29: 1378-1384.

13. Netland PA; Ophthalmic Technology Assessment Committee Glaucoma Panel, American Academy of Ophthalmology. Nonpenetrating glaucoma surgery. Ophthalmology 2001; 108: 416-421.

14. Gianoli F, Schnyder CC, Bovey E, Mermoud A. Combined surgery for cataract and glaucoma: phacoemulsification and 
deep sclerectomy compared with phacoemulsification and trabeculectomy. J Cataract Refract Surg 1999; 25: 340-346.

15. Wishart PK, Wishart MS, Porooshani H. Viscocanalostomy and deep sclerectomy for the surgical treatment of glaucoma: a longterm follow-up. Acta Ophthalmol Scand 2003; 81: 343-348.

16. Bove G, Kantelip B, Algros MP. La sclerectomie profonde avec trabeculectomie externe: un formidable pari! $J \mathrm{Fr}$ Ophtalmol 2001; 24: 332-333.
17. Sanchez E, Schnyder CC, Sickenberg M, Chiou AG, Hediguer SE, Mermoud A. Deep sclerectomy: results with and without collagen implant. International Ophthalmology 1997; 20: 157-162.

18. Manelli A. El manejo postoperatorio de la cirugía filtrante no perforante. Goniopunción con Láser Nd:Yag. In: Rebolleda G, Muñoz Negrete FJ. Manejo postoperatorio de la cirugía filtrante y sus complicaciones. Madrid: Rigorma Gráfica S.A; 2001; 183-188. 